\title{
TRASTORNOS DE LA FERTILIDAD EN LA MUJER CON DIABETES TIPO 1 Y $2^{1}$
}

\author{
FERTILITY DISORDERS IN WOMEN \\ WITH TYPE 1 AND $2^{1}$ DIABETES
}

Carolina Gómez Martín, Natalia Maccio (coordinadoras), Marisa Geller (experta invitada). Integrantes: Celina Bertona, Julia Migueles, Bárbara Arinovich, Ana Schindler, Natalia Garrido, Soraya Larreburo, Mónica Blanco, Santiago de Loredo, María Laura Pomares, Lázaro González

A) Epidemiología

B) Etiopatogenia. Causas de infertilidad en la mujer con diabetes.

1) Daños directos producidos por las alteraciones endocrino-metabólicas:
a. Efectos de los niveles de insulina sobre la secreción de gonadotrofinas.
b. Efectos de la insulina sobre la foliculogénesis.
c. Control metabólico y ovulación.

2) Daños secundarios a neuropatía y/o vasculopatía.

3) Síndrome de ovario poliquístico y diabetes.

4) Obesidad-insulinorresistencia e infertilidad.

5) Otras:

- Presencia de otras enfermedades autoinmunes.

- Mayor frecuencia de infecciones vaginales y enfermedad pélvica inflamatoria, vejiga neurogénica.

C) Causas de infertilidad más frecuentes en las distintas etapas de la vida en pacientes con diabetes tipo 1 y 2

- Niñez.

- Adolescencia.

- Adultez.

\footnotetext{
${ }^{1}$ XV Jornada de Graduados en Diabetes 2013. Comité de Graduados. Sociedad Argentina de Diabetes. Tema: Alteraciones de la fertilidad en la persona con diabetes mellitus

Coordinadora: María Laura Pomares, Médica Especialista en Nutrición (UBA), Especializada en Diabetes (SAD). Hospital Pediátrico Juan Pablo II

Secretario: Alejandro de Dios

Contacto del autor: María Laura Pomares

E-mail: pomares1@hotmail.com

Correspondencia: Centro Médico Cegym. Av. San Martín 569, Corrientes, Corrientes (CP 3400), Argentina. Tel.: 4429254.

Móvil: 3794-753187

Fecha de trabajo recibido: 1/4/14

Fecha de trabajo aceptado:16/4/14
}

D) Implicancia de otros factores de riesgo cardiovascular sobre la fertilidad en la mujer

- Tabaquismo.

- Alcohol.

- Dislipemia.

- HTA.

E) Drogas orales utilizadas para el tratamiento de la diabetes y su impacto sobre la fertilidad en la mujer

F) Drogas empleadas para el tratamiento de la infertilidad y su impacto sobre el control metabólico

\section{INTRODUCCIÓN}

La fertilidad es la capacidad fisiológica de una mujer, de un hombre o de una pareja para producir un hijo vivo. Son múltiples los factores que pueden interferir en este complejo proceso. La diabetes mellitus -como enfermedad crónica que afecta el metabolismo- puede alterar dicho proceso en sus distintas etapas. En la siguiente revisión analizamos las evidencias existentes acerca de los distintos aspectos que relacionan la diabetes con las alteraciones de la fertilidad femenina.

\section{A) Epidemiología}

Antes del inicio de la insulinoterapia, la infertilidad era muy frecuente en las mujeres con diabetes tipo 1 (DM1) y cuando el embarazo se producía, la mortalidad fetal y neonatal alcanzaba el 60\%. El manejo intensivo materno fetal, los avances en la insulinoterapia y los cuidados neonatales redujeron esta cifra del 2 al 5\%?. El estudio más importante que evaluó específicamente la fertilidad en pacientes con diabetes fue un estudio de cohorte realizado en Suecia ${ }^{2}$, donde se 
evaluaron 5.978 pacientes con DM1 diagnosticadas antes de los 16 años desde 1965 a 2004, con un seguimiento de $13,3 \pm 8,9$ años, y se registró una reducción del $20 \%$ en la tasa de nacidos vivos respecto de la tasa esperada por edad en la población femenina general. Cuando se compararon las pacientes que fueron diagnosticadas antes y después del año 1985, el diagnóstico previo a dicho año se correlacionó con un mayor número de complicaciones micro y macrovasculares y menor fertilidad ${ }^{2}$. En las mujeres con diabetes tipo 2 (DM2), la infertilidad se asoció fuertemente a la presencia de síndrome de ovario poliquístico. Conn et al. evaluaron a 49 mujeres con DM2 y encontraron ovarios poliquísticos en la ecografía en el $82 \%$ de los casos; de ellas, el 52\% tenía manifestaciones clínicas de hiperandrogenismo y/o irregularidades menstruales ${ }^{3}$. Por otra parte, la presencia de oligomenorrea aumentó el riesgo de padecer DM2 en el futuro. En el Nurse's Health Study II, un estudio observacional de cohorte, se evaluaron 101.073 mujeres sin historia previa de diabetes; aquellas con ciclos menstruales mayores a 40 días entre los 18 y 22 años tuvieron un riesgo relativo de 2,08 de desarrollar DM2, independiente del IMC4. Similares resultados observaron Shim et al. en mujeres coreanas, postulando que la DM2, las irregularidades menstruales y la infertilidad se asocian fuertemente porque comparten importantes mecanismos fisiopatogénicos: obesidad central e hiperinsulinemia ${ }^{5}$.

\section{B) Etiopatogenia}

1. Daños directos producidos por alteraciones endocrino-metabólicas

\section{a. Efectos de los niveles de insulina sobre la secreción de gonadotrofinas}

Diversos estudios de los efectos metabólicos de la DM1 y DM2 sobre el eje hipotálamohipófiso-gonadal $(\mathrm{HHG})$ demostraron la presencia de alteraciones menstruales, como retraso de la menarca, alteraciones del ritmo menstrual y falla ovárica prematura, y sus consecuencias sobre la fertilidad. Hasta un $40 \%$ de las mujeres con DM1 presenta alguno de estos trastornos en el transcurso de vida ${ }^{6}$. La causa más frecuente de amenorrea en estas pacientes es la amenorrea funcional sin alteraciones del eje $\mathrm{HHG}^{7}$. Los problemas reproductivos en las pacientes con DM1 han presentado significativos cambios con los avances en el tratamiento. Previo al uso de insulina, se observaban hipogonadismo severo y muy bajos niveles de fertilidad. Después de la introducción de la insulina, con el tratamiento convencional de dos dosis de insulina al día, los ciclos menstruales y la fertilidad mejoraron pero persistieron las amenorreas primarias y secundarias, además del marcado retraso puberal por insulinización insuficiente $^{6}$. Luego de la publicación del Diabetes Control and Complication Trial ${ }^{8}$, que marcó el inicio de los tratamientos intensificados, comenzaron a evidenciarse los trastornos por el reemplazo no fisiológico de insulina, asociado al control glucémico sub-óptimo en algunos casos (Figura 1).

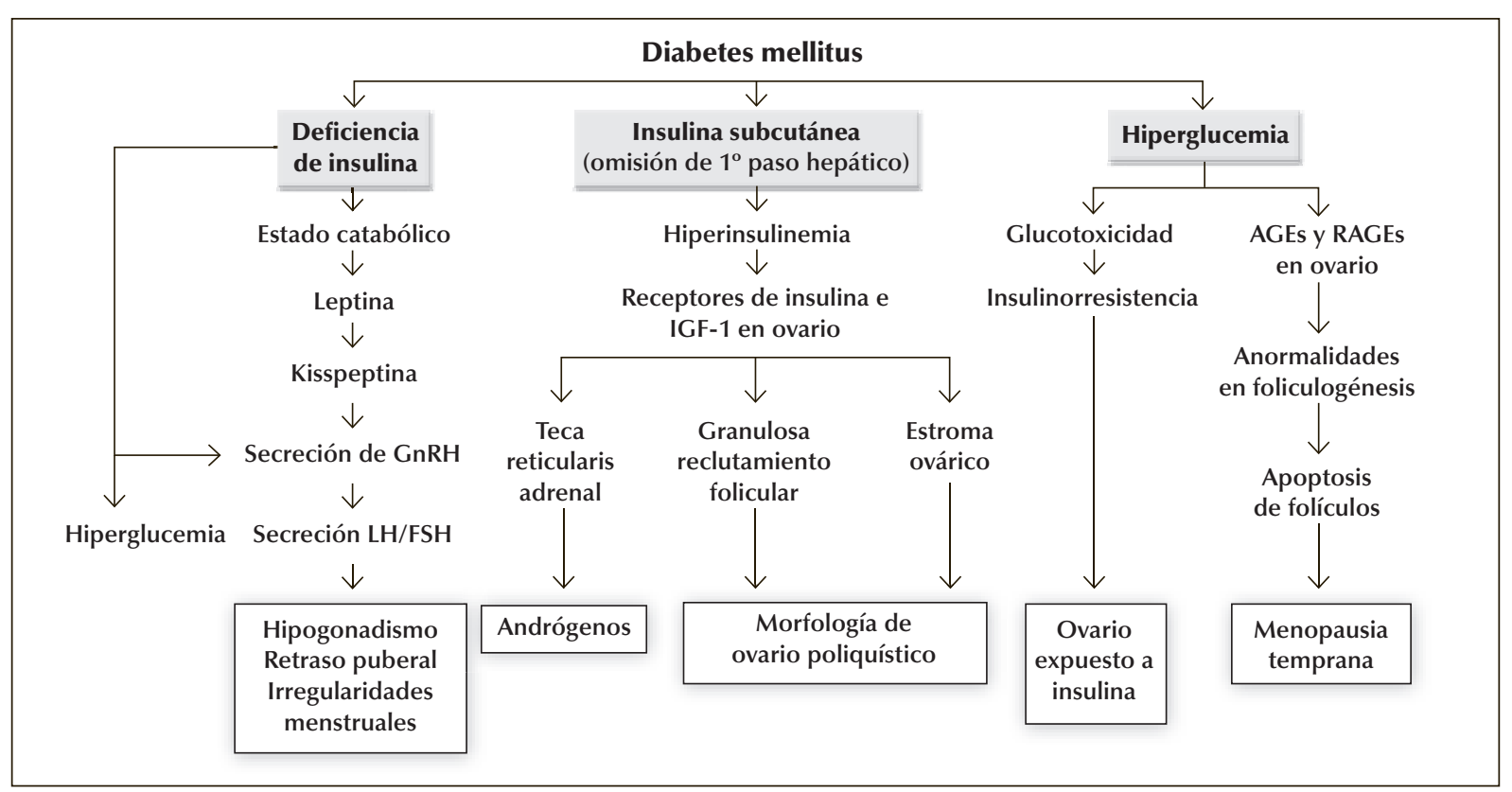

Figura 1: Adaptado de Codner, E. Reproducción femenina y diabetes tipo 1: desde los mecanismos a los hallazgos clínicos. Human Reproduction Update, Vol. 18, Nº 5 pp. 568-585, 2012 
La secreción pulsátil de GnRH estimula la secreción de LH y FSH en la hipófisis, que son las hormonas reguladoras del desarrollo y la función gonadal. Las kisspeptinas (productos del gen Kiss I, sintetizadas por neuronas del hipotálamo) son reguladoras centrales de la pubertad y de la reproducción dado que activan a las neuronas que sintetizan $\mathrm{GnRH}$. Los niveles bajos de leptina, secundarios a pérdida de peso por insuficiente insulinización, reducirían la actividad de las neuronas productoras de kisspeptinas, disminuyendo su estímulo sobre las neuronas $\mathrm{GnRH}^{6}$ (Figura 1). Por su parte, la insulina podría regular directamente la secreción de $\mathrm{GnRH}$ : modelos animales con deleción específica del receptor de insulina en neuronas desarrollaron severas alteraciones metabólicas, hipogonadismo hipogonadotrófico e infertilidad ${ }^{9}$. En mujeres con DM1, con mal control metabólico, se produjo un hipogonadismo hipogonadotrófico similar al asociado a otras formas de estrés metabólico, como la anorexia nerviosa, presentando niveles bajos de FSH, $\mathrm{LH}$ y estradiol ${ }^{7}$ (Figura 1). La mayoría de los estudios demuestra una normal respuesta a la administración exógena de $\mathrm{GnRH}$, reforzando la hipótesis de que el hipogonadismo es secundario a las alteraciones hipotalámicas ${ }^{10}$. Otros autores postulan un efecto tóxico directo de la hiperglucemia sobre las neuronas hipotalámicas ${ }^{11}$. Arrais y Dib sostienen que otros mediadores como dopamina, opioides y catecolaminas podrían estar involucrados en el hipogonadismo de estas pacientes?

\section{b. Efectos de la insulina sobre la foliculogénesis}

Con el advenimiento de los tratamientos intensificados en DM1, como se mencionó anteriormente, se produjo un incremento de las anormalidades en la fertilidad debido al exceso de insulina, especialmente hiperandrogenismo, ovarios poliquísticos y excesiva ganancia de peso ${ }^{6}$. Cuando la insulina se aplica en el tejido subcutáneo, se absorbe a la circulación sistémica, omitiendo el primer paso hepático y exponiendo a los tejidos (entre ellos, el ovario) a niveles suprafisiológicos. La importancia de la insulina en la función reproductiva se pone en evidencia por la presencia de receptores de insulina (hipotálamo, hipófisis, útero y ovarios) e IGF 1 (ovarios) $^{12}$. Actuando sobre las células de la granulosa, la insulina estimula el desarrollo folicular, aumentando el reclutamiento y crecimiento folicular preovulatorio ${ }^{13}$; reduce la apoptosis y atresia de folículos ${ }^{14}$, y promueve el crecimiento ovárico. Codner et al. observaron mayor volumen ovárico y mayor número de folículos de gran tamaño en adultas con $D M 1^{15}$ y la formación de quistes. Además, potencia la síntesis de estrógenos estimulada por FSH lactúa como co-gonadotrofina) ${ }^{13}$. Sobre las células de la teca, aumenta la actividad de las enzimas esteroideogénicas (CYP17, p450scc, 3ß HSD) ${ }^{13}$ y estimula la secreción de andrógenos (actúa como co-gonadotrofina con LH). Algunas mujeres con DM1 podrían tener una respuesta anormal a la insulina exógena, presentando hiperandrogenismo ante niveles normales de insulina ${ }^{12}$.

\section{c. Control metabólico y ovulación}

La hiperglucemia per se es otro factor que altera la fertilidad. Niveles glucémicos elevados inducen aumento de la resistencia periférica a la insulina que, asociada a la hiperinsulinemia existente, conducen al hiperandrogenismo y poliquistosis ovárica, aún en pacientes con $\mathrm{DM}^{16}$. Por otra parte, estudios en ratas diabéticas por estreptozotocina mostraron la reducción de folículos preovulatorios y retraso en la maduración del ovocito, con incremento de la apoptosis ${ }^{16}$.

Se demostró expresión de receptores productos avanzados de la glicosilación en células de la teca y la granulosa. En pacientes con síndrome de ovario poliquístico, se indicó mayor expresión de dichos receptores en células de la granulosa que en aquellas normales ${ }^{17}$.

Respecto del control glucémico y la ovulación, Codner et al. evaluaron ciclos ovulatorios en adolescentes con DM1 y controles. El promedio general no mostró diferencias. Pero cuando se analizaron pacientes según el nivel de $\mathrm{HbA} 1 \mathrm{c}$, aquellas con $\mathrm{HbA} 1 \mathrm{c}$ mayor a 7,5\% tuvieron un porcentaje menor de ciclos ovulatorios $(p=0,012)^{18}$.

\section{Daños secundarios a neuropatía y/o vasculopatía}

Pocos trabajos se encuentran en la literatura que evalúen específicamente la asociación de las alteraciones de la fertilidad con la neuropatía y vasculopatía. El primer estudio epidemiológico sobre fertilidad en pacientes con DM1 (el sueco mencionado anteriormente) dio como resultado que las mujeres con DM1 eran un 20\% menos fértiles respecto de la población femenina general, utilizando una tasa de fertilidad estandarizada 
(TFE=0,80). La presencia de complicaciones crónicas se asoció con una menor tasa de fertilidad. En la Tabla 1 se observa la TFE según presencia o no de complicaciones. La enfermedad cardiovascular es la complicación que se asoció a una menor fertilidad (reducción del $66 \%$ de la tasa de fertilidad vs. $20 \%$ en pacientes sin complicaciones) ${ }^{2}$.

\begin{tabular}{|l|c|c|}
\hline Complicación & Ausente & Presente \\
\hline Retinopatía & 0,84 & 0,63 \\
\hline Nefropatía & 0,82 & 0,54 \\
\hline Neuropatía & 0,81 & 0,50 \\
\hline Cardiovascular & 0,80 & 0,34 \\
\hline
\end{tabular}

Tabla 1: Tasa de fertilidad estandarizada (TFE) entre mujeres entre mujeres DM1 estadificadas según tipo de complicaciones.

En una revisión sobre los mecanismos que generan las complicaciones crónicas de la diabetes y las alteraciones de la función sexual, Amaral S. et al. postulan que el estado de hiperglucemia crónica genera la superproducción de especies reactivas de oxígeno (ERO) que, asociada a la reducción de los mecanismos defensivos antioxidantes, desencadenaría daño mitocondrial severo y podría ser disparador de varias alteraciones de la función sexual. La excesiva producción de ERO afectaría diferentes etapas de la embriogénesis y del desarrollo del embarazo $^{19}$. Por otra parte, se sugiere que el estrés oxidativo regularía el declive de la fertilidad femenina en relación con la edad ${ }^{20,21}$. Las ERO afectan la maduración del ovocito, el desarrollo del embrión y del embarazo (por disfunción mitocondrial) ${ }^{22}$.

En modelos animales se demostró que la diabetes provoca disfunción del ovocito por envejecimiento precoz (disminución del óxido nítrico), contribuyendo así a fallo reproductivo, aberraciones cromosómicas e infertilidad ${ }^{23}$. También se comprobó sobreproducción de agentes proinflamatorios en el útero, durante la implantación y desarrollo del embrión y placenta, que activaría metaloproteinasas y proteasas en la matriz uterina, implicadas en la remodelación de la matriz extracelular durante la implantación y el desarrollo del feto-placentaria ${ }^{24}$.

\section{Síndrome de ovario poliquístico y diabetes}

El síndrome del ovario poliquístico (SOP) es un desorden heterogéneo, endocrino-metabólico, reconocido como la principal causa de infertilidad por anovulación en mujeres. Afecta a entre el 5-10\% de las mujeres en edad reproductiva ${ }^{25}$. Ensayos clínicos prospectivos demostraron una prevalencia del 30$35 \%$ de intolerancia a la glucosa y del 7,5 al $10 \%$ de DM2 en mujeres con SOP26. Debido a su heterogeneidad, no existe un acuerdo universal en cuanto a los criterios diagnósticos. El amplio espectro de síntomas y signos de este síndrome incluye trastornos menstruales, hirsutismo, acné, obesidad y ovarios poliquísticos por ecografía, y exige la exclusión de otras patologías. Su etiología aún es controversial, pero existen evidencias de su origen multifactorial con fuerte influencia genética asociada, por un lado, a una alteración a nivel del eje hipotálamo-hipofisario-ovárico con mayor síntesis de andrógenos, y por el otro, a insulinorresistencia con hiperinsulinemia ${ }^{27}$. Además, la conversión de intolerancia a la glucosa a diabetes aumenta de 5 a 10 veces en estas pacientes. En aquellas con DM1, la administración de dosis suprafisiológicas de insulina resulta en una estimulación de la síntesis de andrógenos aumentada, como ocurre en los estados de insulinorresistencia ${ }^{28}$. Existen descripciones de prevalencia de SOP en mujeres con DM1 que van desde el 18,8 hasta un $40,5 \%$, según el criterio diagnóstico utilizado ${ }^{29}$. La anovulación es la causa más frecuente de infertilidad ${ }^{30}$, siendo el SOP el responsable del $90 \%$ de los casos ${ }^{31}$. De las mujeres con infertilidad y SOP, aproximadamente entre el $80-90 \%$ presenta sobrepeso. La obesidad agrava de forma independiente la fertilidad ${ }^{32}$. Datos latinoamericanos reportan una incidencia de aborto de entre el 30-50\% de las pacientes con SOP que se embarazan. A su vez, más del $30 \%$ de las abortadoras habituales tiene SOP${ }^{33}$. Las mujeres embarazadas con SOP experimentan una mayor incidencia de morbilidad perinatal y sus bebés están en mayor riesgo de complicaciones neonatales. Se evidencian las siguientes alteraciones endocrinas (Figura 2) ${ }^{34}$ :

Nivel hipotalámico

- Aumento de la frecuencia de secreción de pulsos de GnRh.

- Disminución de sensibilidad del generador de GnRh (anormalidad intrínseca del generador o bajo nivel de progesterona?).

Nivel hipofisario

- > frecuencia y amplitud de LH.

- Bajos niveles de FSH.

Nivel ovárico

- Esteroidogénesis aumentada en la teca.

- > eficiencia en conversión de andrógenos.

- Amplificados por hiperestimulación de LHInsulina.

- Mecanismo de retrocontrol atenuado. 


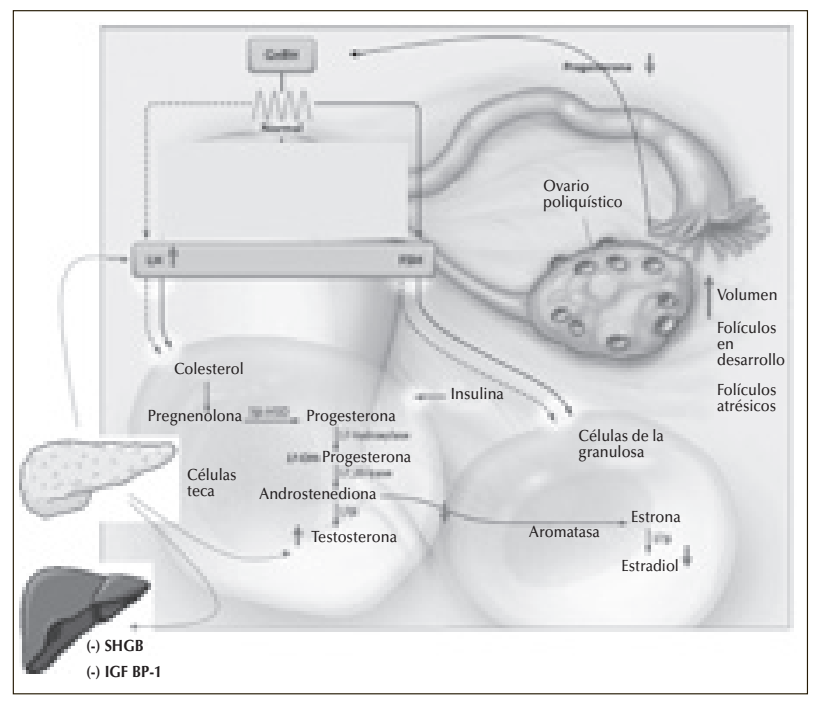

Figura 2: Alteraciones endocrinas en el síndrome de ovario poliquístico (SOP). Adaptado. Adaptado de: Ehrmann D A. Polycystic Ovary Syndrome. N. Eng.I J. Med. 352:1223-1236, 2005.

Mecanismos de alteración de la fertilidad en SOP35

- Anovulación.

- Alteración de la calidad del ovocito y del embrión.

- Alteraciones endometriales y de la decidualización: disminuye la glicodelina IGFBP-1 del endometrio, aumenta la actividad de PAI-1, disminuye la vasculatura uterina, aumenta la resistencia vascular uterina.

- Alteraciones placentarias: disminución del grosor, densidad y volumen, con aumento de la forma irregular y del índice de plegamiento del cordón umbilical.

\section{Obesidad-insulinorresistencia e infertilidad}

La obesidad es una enfermedad multifactorial que incluye factores exógenos y endógenos y se ha convertido en una epidemia mundial ${ }^{36}$. El tejido adiposo es el órgano endocrino más grande del cuerpo humano, con efectos sobre la homeostasis de la glucosa, la producción de esteroides, el sistema inmune y la función reproductiva ${ }^{37}$.

La anovulación y la infertilidad ocurren con mayor frecuencia en mujeres obesas comparadas con aquellas de peso normal ${ }^{38}$.

La conexión estrecha entre la obesidad y la insulinorresistencia es la respuesta inflamatoria. La obesidad es un estado de inflamación crónico marcado por la invasión de macrófagos dentro del tejido adiposo y por el incremento de citoquinas proinflamatorias que no se evidencian en los individuos delgados $^{39}$. Los receptores de insulina y los factores insulinoides de crecimiento pertenecen a la familia de receptores tirosina-cinasa. La insulina estimula la fosforilación tirosina del IRS. Este paso de señales insulina-receptor es defectuoso en la mayor parte de los casos con insulinorresistencia, inhibiendo la acción de la insulina por la fosforilación serina del IRS-1 e interrumpiendo la señal insulina-receptor ${ }^{40}$.

El oxígeno es esencial para la génesis máxima de energía pero puede dañar la célula mediante formas diversas de estrés oxidativo. A partir de éste, comienzan a modificarse adversamente la integración de señales bioquímicas ${ }^{41}$.

La prevalencia de resistencia a la insulina en pacientes con infertilidad es de 50-60\%.

A nivel ovárico, los receptores de insulina se distribuyen en todos los compartimientos. La insulina estimula y aumenta la secreción de estrógenos, progesterona y andrógenos. Se ha demostrado elevación de androstenediona y dehidroepiandrostenediona en mujeres obesas con resistencia a la insulina, produciendo hiperandrogenismo y, por lo tanto, anovulación ${ }^{42}$.

Existen numerosos factores neuroendocrinos encargados de regular el metabolismo energético, el más importante es la leptina. Es secretada y sintetizada en el tejido adiposo como respuesta a la alimentación para así suprimir el apetito a través de una vía hipotalámica. Ejerce su efecto al disminuir la ingesta y, por lo tanto, el peso corporal, aumentando la oxidación de grasa y el gasto energético ${ }^{43}$. Los estrógenos estimulan la producción de leptina por el tejido adiposo en las mujeres, pero no en los hombres. La leptina ha sido encontrada en el líquido perifolicular y sus receptores han sido localizados en el folículo preovulatorio y el ovocito maduro, jugando un papel primordial en la etapa ovulatoria ${ }^{44}$. Además juega un rol importante en el desarrollo embriológico, dado que sus receptores han sido localizados en embriones preimplantados. Los niveles plasmáticos se encuentran elevados tanto en plasma materno como fetal, durante el período de embarazo ${ }^{45}$. En conclusión, la leptina es una señal periférica de adecuada energía, requerida para una regulación normal del peso corporal, insulino sensibilidad y funciones reproductiva y neuroendocrina adecuadas ${ }^{46}$.

La obesidad se asocia con resistencia a la leptina como a su déficit. Los bajos niveles de leptina o su deficiencia en la acción generan un círculo vicioso de hiperinsulinemia, insulinorresistencia, hipercortiso- 
lismo, hipotiroidismo, bajos niveles de hormonas reproductivas, infertilidad y obesidad, observándose ${ }^{47}$ :

- Hiperfagia.

- Incremento de lipogénesis central con acumulación de triglicéridos en el tejido adiposo y de la lipólisis periférica.

- Disminución en la termogénesis.

- Atrofia uterina y ovárica, hipogonadismo, ciclos menstruales y estrogénicos anormales, oligoanovulación y deterioro en la función y morfología de la glándula mamaria ${ }^{48}$.

- Disminución de la hormona de crecimiento, esencial para la normal función reproductiva, resultando en menor desarrollo puberal e infertilidad ${ }^{49}$.

La administración de leptina en mujeres durante dos meses incrementó los niveles de leptina con asociación de ovulación normal, mejora en la morfología endometrial y ovárica, incluso en la función hipotalámica.

Otras adipoquinas han sido involucradas en relación a la obesidad e infertilidad:

- La baja expresión de adiponectina asociada a insulinorresistencia interfiere con la foliculogénesis y la secreción de esteroides.

- Los niveles aumentados de IL-6 también incrementan la insulinorresistencia y deterioran la secreción de LH.

- El incremento de TNF- $\alpha$ reduce la insulinosensibilidad, inhibe la secreción de gonadotrofinas, induce la regresión del cuerpo lúteo y deteriora el desarrollo endometrial ${ }^{50}$.

- Los niveles plasmáticos de ácidos grasos libres (AGL) son elevados en obesidad y causan una disminución en la captación periférica de glucosa y un incremento en la producción hepática de glucosa. Además disminuyen las células de la granulosa por apoptosis contribuyendo a la anovulación ${ }^{51}$ e incrementan el pool de andrógenos aumentando la producción de precursores androgénicos de las glándulas adrenales directamente y no a través del mecanismo central (ACTH).

Las medidas no farmacológicas como ejercicio, pérdida de peso del $5 \%$ o más y hábitos alimentarios saludables, disminuyen la hiperinsulinemia, el hiperandrogenismo, la insulinorresistencia y aumentan los niveles de leptina, permitiendo la recuperación del ciclo ovulatorio y la normalización del ciclo endocrino metabólico ${ }^{52}$. Múltiples estudios han demostrado que luego de la cirugía bariátrica y la consecuente pérdida de peso, se obtienen los mismos resultados y las tasas de fertilidad mejoran ${ }^{53}$.

\section{Otras causas de infertilidad. Autoinmunidad}

Se ha reportado una prevalencia de más del $65 \%$ de autoanticuerpos dirigidos contra al menos uno de los tejidos de ovario analizados en las mujeres jóvenes con DM1 en comparación con las no diabéticas $(4,8 \%)$. La ooforitis autoinmune puede ocurrir como parte de los síndromes de insuficiencia poliglandular autoinmune tipo I y tipo II. También se describe la tiroiditis autoinmune en un porcentaje mayor al $70 \%$ de las pacientes con irregularidades menstruales. Aquellas con DM1, que tienen hipotiroidismo manifiesto o subclínico, también pueden estar en riesgo de insuficiencia ovárica prematura. En general, la evaluación de la insuficiencia ovárica prematura debe incluir la concentración sérica de FSH y estradiol, además de la detección de la insuficiencia adrenal autoinmune asintomática, dado que es más común en mujeres con insuficiencia ovárica autoinmune.

En el ciclo menstrual se observan cambios en las pacientes con tiroiditis de Hashimoto que incluyen menorragia, períodos más largos y más frecuentes, y dismenorrea. La prevalencia de autoinmunidad tiroidea es significativamente mayor en las mujeres infértiles, especialmente entre aquellas en las cuales la infertilidad es causada por endometriosis o disfunción ovárica9 .

\section{Enfermedad celíaca}

En las mujeres celíacas la menarca es tardía y la menopausia más temprana acortando el período de fertilidad; la tasa de abortos es mayor y aumentan las complicaciones durante el embarazo respecto de aquellas que cumplen una dieta libre de gluten. La patogénesis de los desórdenes en el eje gonadal asociados a la enfermedad celíaca aún no ha sido resuelta. Una hipótesis es que el gluten actuaría como autoantígeno, generando anticuerpos contra órganos y hormonas involucrados en el desarrollo puberal. Otra hipótesis sería que la falta de micronutrientes secundaria a la malabsorción tendría como consecuencia alteraciones en la SHBG (proteína transportadora de hormonas sexuales) y en los receptores proteicos. El zinc, el cobre y el selenio cumplirían determinadas funciones en el desarrollo puberal y en la secreción de GH, FSH y LH. La amenorrea está directamente relacionada con el peso corporal. El peso corporal promedio a la edad de la menarca es de $47,5 \mathrm{~kg}^{5}$. Cuando los depósitos de grasa son bajos, disminuye la lepti- 
na $y$, en consecuencia, aumenta el apetito lo cual contribuye al aumento de peso y estimula la secreción de $\mathrm{GnRH}$ a través de un mecanismo indirecto, probablemente mediante el neuropéptido $Y$ secretado por las células de la glía. Su concentración es directamente proporcional a la masa grasa. Las concentraciones de leptina aumentan durante la pubertad, por lo cual la hipoleptinemia secundaria al bajo peso estaría involucrada en el hipogonadismo hipogonadotrófico que suele apreciarse en las pacientes con celiaquía y retraso puberal.

\section{Disfunción sexual y diabetes}

El efecto de la diabetes sobre la función sexual femenina no es plenamente reconocido y abordado. Se documenta que la prevalencia de problemas de excitación sexual y lubricación inadecuada existe en el 14 al 45\% entre las mujeres diabéticas, y es significativamente mayor que en los controles sanos. La disfunción sexual femenina puede tener un efecto secundario sobre la fertilidad al disminuir el deseo y la limitación de la actividad sexual, principalmente en la época de ovulación ${ }^{9}$.

\section{C) Causas de infertilidad más frecuentes en las distintas etapas de la vida en pacien- tes con diabetes tipo 1 y 2}

Al dividir el ciclo femenino en tres etapas evolutivas, se observan diferentes causas de infertilidad.

\section{Niñez y pubertad}

- Menarca tardía. El desarrollo de DM1 antes de los 10 años se asocia con esta alteración. Varias décadas atrás, las niñas con DM1 presentaban severo retraso puberal, asociado principalmente al mal control metabólico. En las décadas de 1940-1950 era común encontrar que la menarca comenzaba dos años más tarde que en la población general y eran seguidas de amenorreas $1^{\circ}$ durante la adolescencia por falta de insulina. Hacia la década de 1990, con la intensificación del tratamiento insulínico, el retraso en la aparición de la menarca se acortó y actualmente pasó a ser de entre dos y nueve meses ${ }^{54}$.

- Anormalidades menstruales. El mal control metabólico en esta etapa se asocia fundamentalmente a oligomenorrea, con ciclos menstruales más largos, mayores a 31 días y menstruaciones más abundantes ${ }^{6,55}$.

- Autoinmunidad. Enfermedades como ooforitis, insuficiencia suprarrenal autoinmune e hipertiroidismo autoinmune, se asocian frecuentemente con infertilidad y deben ser evaluadas siempre en niñas diabéticas con alteraciones del ciclo, mediante la pesquisa de auto-anticuerpos anti-ováricos y anticuerpos anti-tiroideos ${ }^{56}$.

\section{Adolescencia}

- Anormalidades menstruales. Estas alteraciones se observan durante las distintas etapas de la vida de la mujer. Pueden dividirse en dos tipos según sus causas:

- Anovulación hipotalámica: la alteración del eje endocrino reproductivo puede ser conceptualizado como la anovulación hipotalámica de la anorexia, dado que en muchas ocasiones la adolescente con DM1 presenta bajo peso y estados catabólicos por restricción alimentaria o falta de niveles adecuados de insulina, que conducen a la depleción intracelular causando, a nivel hipotalámico, alteraciones de la secreción pulsátil de gonadotrofina y ciclos anovulatorios.

- Anovulación, hiperandrogenismo y síndrome de ovario poliquístico: en la actualidad es frecuente encontrar estas alteraciones debido a la ganancia de peso posterior a la menarca que se observa tanto en las adolescentes con DM1 con buen control metabólico y con tratamientos intensificados de insulina, como en aquellas con DM2 e insulinorresistencia ${ }^{6}$.

\section{Adultez}

- Infecciones del tracto genital. Al igual que en la población general, las infecciones del tracto genital pueden ser causales de infertilidad y es común encontrarlas asociadas al mal control metabólico, sepsis vaginales, lesiones tubarias y EPI.

- Disfunción sexual. Si bien esta complicación en la mujer no ha sido tan bien estudiada como en el hombre, en las féminas con diabetes se describen: disminución del deseo sexual, de la excitación, inadecuada lubricación y dispareunias, todas asociadas varias veces a síntomas depresivos ${ }^{57}$.

- Disminución del número de embarazos. Relacionado con el control metabólico, la presencia de complicaciones crónicas y varias veces con las elecciones de estas pacientes de tener un menor número de embarazos por lo difícil que resulta un óptimo control del mismo.

- Menor número de niños nacidos vivos. También muy vinculado con el control metabólico y las complicaciones crónicas.

- Tratamiento de fertilidad. El éxito de estos tratamientos se relaciona con el buen control me- 
tabólico. Las pacientes diabéticas compensadas que se someten a tratamientos de fertilización in vitro tienen los mismos resultados obtenidos que en la población general.

- Menopausia precoz. Se ha descripto la falla ovárica prematura de estas pacientes relacionándola con glucotoxicidad ovárica, así como también con la asociación de complicaciones crónicas, principalmente nefropatía avanzada y retinopatía proliferativa. Algunos estudios señalan la aparición de la menopausia 10 años antes que en la población general, haciendo que estas pacientes tengan períodos reproductivos más cortos ${ }^{58}$.

\section{D) Implicancia de otros factores de riesgo car- diovascular sobre la fertilidad en la mujer Tabaquismo (TBQ)}

En Estados Unidos, el $13 \%$ de los casos de infertilidad femenina se relaciona con el cigarrillo. Diversos trabajos demostraron que las mujeres tabaquistas presentan un mayor tiempo hasta la concepción que aquellas que no fuman ${ }^{59,60}$. El daño del mismo será dosis-dependiente ante quienes consumen más de 10 cigarrillos/día61. Los principales componentes responsables de su toxicidad son la nicotina y el benzo $\alpha$ pireno, pues promueven la formación de radicales libres (ROS) ${ }^{62}$. EI TBO afectaría principalmente la función y la reserva ovárica. Se halló mayor peroxidación lipídica y menor capacidad antioxidante en los folículos ${ }^{63}$. Otros mecanismos posibles serían cambios tubáricos, cervicales, embarazos ectópicos y abortos espontáneos ${ }^{24}$. El TBQ pasivo también demostró tener consecuencias deletéreas similares ${ }^{64}$. Finalmente, el efecto negativo del TBO sobre la fertilidad femenina sería transitorio, pues revertiría luego del abandono del hábito ${ }^{65}$.

\section{Alcohol (OH)}

Si bien diversos estudios relacionan la ingesta de alcohol con disminución de la fertilidad femenina, el nivel de consumo asociado al mayor riesgo no ha sido claramente definido. Un estudio danés halló aumento del riesgo superadas las siete medidas semanales ${ }^{66}$. Más aún, Hakim et al. observaron un incremento en el riesgo de infertilidad incluso con mínimos niveles de consumo ${ }^{67}$. El consumo de $\mathrm{OH}$ se ha relacionado con un mayor tiempo hasta lograr el embarazo ${ }^{20}$ y con una mayor tasa de abortos espontáneos ${ }^{68}$. Se postula que el $\mathrm{OH}$ elevaría los niveles de estrógenos, lo que a su vez conduciría a una inhibición de la FSH afectando, de este modo, la foliculogénesis y la ovulación. Además, ejercería un efecto directo en la maduración del óvulo, la ovulación, el desarrollo del blastocito y la implantación ${ }^{20}$. Un mecanismo de daño propuesto sería la producción de ROS (el consumo regular incrementa su producción y desciende el nivel de enzimas antioxidantes) y la formación de productos de glicosilación avanzada ${ }^{14}$.

\section{E) Drogas orales utilizadas para el trata- miento de la diabetes y su impacto sobre la fertilidad en la mujer}

\section{Metformina (MTF)}

Abundante (y a veces contradictoria) es la bibliografía existente en relación a la metformina (MTF) y la reproducción en la mujer. Dentro de los cambios hormonales inducidos por MTF que podrían modular la fertilidad, se describen el descenso de testosterona total sérica ${ }^{69,70}$, cuya disminución no parecería deberse a cambios en el nivel de $\mathrm{SHBG}^{7}$, y la mejoría de la insulinorresistencia ${ }^{71}$, con reducción de la insulinemia de ayuno (siendo significativo en no obesas, pero no en obesas con SOP). Otros efectos posibles para la droga en relación a la fertilidad femenina son el descenso tanto de hormona antimulleriana $^{72}$ (sustancia que suele estar aumentada en el SOP y se relaciona con la cantidad y calidad de la foliculogénesis) como del inhibidor del activador tisular del plasminógeno (sustancia potencialmente implicada en la posibilidad de aborto).

En relación a la inducción de ovulación, al comparar MTF con placebo, en el metaanálisis de 2012, de Tang et al. ${ }^{73}$ observaron con MTF una mejoría en la ovulación, estadísticamente significativa que, sin embargo, se tornaba no significativa al lograr una heterogeneidad aceptable mediante la realización de un análisis por subgrupos según IMC. Sinawat et al. ${ }^{74}$, en una revisión de Cochrane, también detectaron que la asociación de MTF y citrato de clomifeno (CC) presentaba mayor efectividad que clomifeno aislado en la inducción de la ovulación en SOP, sin poder determinar, con los datos existentes, si la eficacia de un curso corto de pretratamiento con MTF (menor a cuatro semanas) era similar a la de un curso largo convencional antes de iniciar la inducción de ovulación con CC.

En relación a la tasa de embarazo, al comparar MTF con placebo, MTF arrojó mejores resultados en el metaanálisis de 2012 de Tang et al. ${ }^{73}$, pero con 
una gran heterogeneidad que sólo se diluía en el análisis del subgrupo de las pacientes obesas, en el que se demostraba una tendencia al beneficio dado por MTF pero no estadísticamente significativa. En el subgrupo de las no obesas, sin lograr disminuir la heterogeneidad en el análisis, persistía la mejoría con MTF. En la revisión de Teede et al. ${ }^{75}$, MTF superó a placebo en el global de mujeres con SOP y en el subgrupo con $\mathrm{IMC}<30$; sin embargo no obtuvieron diferencia a favor de la droga en los subgrupos de IMC> 30, mujeres naive a CC, resistentes o no a CC.

Con respecto a la tasa de nacimientos, en ninguno de los metaanálisis ni trabajos analizados, MTF logró demostrar una mejoría estadísticamente significativa en comparación con placebo ${ }^{73,75,76}$.

En relación a la tasa de abortos, al compararla con placebo, tanto en el metaanálisis de Tang et al. ${ }^{73}$ como en la revisión de Teede et al..$^{75}$, no se hallaron diferencias significativas a favor de MTF. En ninguno de los trabajos analizados se logró demostrar una diferencia significativa al comparar MTF y CC como drogas aisladas, así como tampoco al comparar la asociación de MTF con CC vs MTF o CC aislados ${ }^{73,75,77,78}$

Dentro de otros efectos evaluados en relación a la fertilidad femenina, Tang et al. informaron que MTF pareció superar a placebo en recomponer el ritmo menstrual.

En conclusión, parecería que MTF podría aumentar las chances de ovulación y embarazo en SOP, asociándola necesariamente a CC en el subgrupo de pacientes obesas para obtener mejores resultados.

Sin embargo, dado que con el uso de MTF no pudo comprobarse un aumento en la tasa de recién nacidos vivos, ni resultó suficientemente eficiente para regularizar los ciclos menstruales y tratar el hiperandrogenismo ${ }^{79}$, no debería ser usada como droga de primera línea en los tratamientos reproductivos. Las indicaciones de MTF en el contexto de fertilidad serían las mismas que para el uso habitual de la droga: tratamiento de mujeres con diabetes o de mujeres con SOP no diabéticas pero con intolerancia a hidratos de carbono o glucemia en ayunas alterada cuando el IMC es > 35 .

\section{Tiazolidinedionas (TZDs)}

Se describen múltiples efectos de las tiazolidinedionas (TZDs) en relación con la fertilidad femenina. Distintos trabajos reconocen con su uso un descenso en los niveles de andrógenos totales y libres (testosterona, DHEA-S, delta 4, con un au- mento de SHBG80), sin embargo, en el metaanálisis de Tang et al. ${ }^{73}$, pioglitazona o rosiglitazona presentaron mínimos efectos o ningún efecto significativo sobre testosterona y SHBG al ser comparadas con placebo o no tratamiento. Si bien no se encuentra confirmada, la posibilidad de una acción directa de las TZDs sobre el ovario no puede descartarse; en células ováricas humanas in vitro se han observado cambios en los niveles de secreción de progesterona, estradiol y testosterona luego del uso de TZDs ${ }^{81}$. En principio, serían las mejoras en el status metabólico, dadas por la redistribución del tejido graso, las que explicarían los cambios en los niveles androgénicos y de estrona circulantes, favoreciendo el retorno a una normal pulsatilidad gonadotrofínica. Tang et al. observaron una mayor regularización del ritmo menstrual con TZDs al compararlas con placebo o no tratamiento ${ }^{82}$. Fromentand et al. ${ }^{83}$ registraron que en distintos trabajos las TZDs resultaban similares o incluso mejores que MTF para el logro de ciclos regulares, y que al comparar la asociación de MTF más pioglitazona, el porcentaje de menstruaciones aumentaba dos veces en comparación con MTF aislada.

Las TZDs podrían inducir la ovulación espontánea, aumentando su tasa en SOP. En comparación con placebo, rosiglitazona presentó un aumento muy significativo de tasa de ovulación ${ }^{82}$.

Existen reportes de casos de embarazos exitosos logrados a partir de la incorporación de TZDs al esquema terapéutico de mujeres con diabetes y SOP ${ }^{44,85}$. Sin embargo, en el metaanálisis de Tang et al. se informó la ausencia de datos disponibles sobre la tasa de embarazo y nacimientos para TZDs en estudios de calidad analizados. Debe, sin embargo, recordarse que pioglitazona y rosiglitazona están catalogadas por la FDA como drogas de categoría C en el embarazo, por lo cual no son utilizadas en el contexto de búsqueda activa de fertilidad.

\section{Acarbosa}

Si bien es una droga poco utilizada, existen trabajos y revisiones que evaluaron su rol en el SOP. Kircher y Smith ${ }^{86}$ publicaron en 2008 una revisión sistemática de trabajos relevantes (con búsqueda desde 1950) sobre acarbosa y SOP. Los estudios hallados presentaban resultados prometedores en cuanto a los posibles beneficios de acarbosa sobre la fertilidad, sin embargo estaban limitados por muestras muy escasas (con $\mathrm{N}$ no mayores de 30 mujeres) y métodos no claramente definidos. 
Con su uso aislado o asociado a citrato de clomifeno, se han observado, entre sus efectos, una disminución en los niveles de insulinemia de ayuno, $\mathrm{LH}$ y andrógenos libres, así como un aumento en la SHBG ${ }^{87,88}$, con mejoría del IMC, de la relación $\mathrm{LH} /$ $\mathrm{FSH}$, el ritmo menstrual y la tasa de ovulación ${ }^{89,90}$.

\section{Sulfonilureas}

En relación a las sulfonilureas no se han hallado, en esta búsqueda bibliográfica, trabajos que estudiaran específicamente sus efectos para mejorar la fertilidad femenina. Se han descripto embarazos exitosos en mujeres que recibían glibenclamida como tratamiento de diabetes monogénicas, al continuar su uso durante la gestación ${ }^{91,92}$.

A la fecha, existe evidencia insuficiente para recomendar el uso de ADOs en mujeres con antecedentes de DMG, tolerancia alterada a la glucosa o DM preexistente que se encuentran en plan de embarazo, o con DM preexistente que cursan embarazos ${ }^{93}$.

\section{Incretinas}

Aún es muy escasa la bibliografía que indaga sobre los efectos de las incretinas en la fertilidad femenina. A partir de estudios con ratones, resultaría necesaria una expresión normal de GLP1 en las células somáticas ováricas para lograr una fertilidad normal en las hembras ${ }^{94}$, como así también se ha observado en esta especie que la inhibición de la DPP 4 suprimiría el aumento de abortos relacionados con estrés y con cambios en la expresión de antígenos de superficie de las células ${ }^{95}$. En placentas humanas de pacientes con preclampsia y RCIU se han objetivado niveles de actividad DPP4 significativamente mayores que en placentas controles ${ }^{96}$, estimándose que la DPP4 placentaria podría tener influencia sobre el metabolismo fetal mediante la degradación de péptidos fetales bioactivos que participan en la regulación del crecimiento intrauterino.

\section{E) Drogas empleadas para el tratamiento de la infertilidad y su impacto sobre el control metabólico}

En toda paciente con diabetes mellitus y alteraciones de la fertilidad resulta fundamental optimizar el control metabólico y promover cambios en el estilo de vida para alcanzar el IMC adecuado antes de indicar drogas para inducir la ovulación. Con las drogas más frecuentemente utilizadas para tal fin -que son el citrato de clomifeno, de primera elección en SOP, y las gonadotrofinas empleadas en la amenorrea hipotalámica funcional y falla ovárica precoz-, no se describieron alteraciones en el metabolismo hidrocarbonado.

\section{CONCLUSIONES}

La diabetes mellitus tipo 1 y 2 deben considerarse en el diagnóstico diferencial de los trastornos menstruales y la infertilidad. El período reproductivo de las mujeres diabéticas puede reducirse debido al retraso de la menarca y la menopausia prematura.

Durante los años reproductivos, la diabetes se ha relacionado con alteraciones menstruales, como oligomenorrea y amenorrea secundaria. Se encontró que un mejor control glucémico y la prevención de las complicaciones diabéticas mejoran estas irregularidades con tasas de fecundidad cercanas a las que se observan en la población general. Las mujeres con trastornos menstruales persistentes, a pesar del tratamiento adecuado, deben ser abordadas con una evaluación más amplia que incluirá: el examen del eje hipotálamo-hipófisis-ovario y el estado hormonal, la presencia de la enfermedad tiroidea autoinmune y autoanticuerpos antiováricos e hiperandrogenismo.

\section{REFERENCIAS}

1. Vargas R, Repke J, Ural S. Type 1 diabetes mellitus and pregnancy. Rev. Obstet. Gynecol. Summer; 3(3): 92-100, 2010.

2. Jonasson J, Brismar K, Sparén P, Lambe M, Nyrén O, Ostenson C, YeW. Fertility in women with type 1 diabetes. A population-based cohort study in Sweden. Diabetes Care 30:2271-2276, 2007.

3. Conn J, Jacobs H, Conway G. The prevalence of polycystic ovaries in women with type 2 diabetes mellitus. Clinical Endocrinology. Vol 52. Issue 1. 81-86, 2000.

4. Solomon C. Long or highly irregular menstrual cycles as a marker for risk of type 2 diabetes mellitus. JAMA 286(19):2421-2426, 2001.

5. Shim U, Oh JY, Lee HJ, Hong YS, Sung YA. Long menstrual cycle is associated with type 2 diabetes mellitus in korean women. Diabetes Metab. J 35:384-389, 2011.

6. Codner E, Merino P,Tena-Sempere M. Female reproduction and type 1 diabetes: from mechanisms to clinical findings. Human Reproduction Update, Vol.18, N 5 pp. 568-585, 2012.

7. Arrais RF, Dib SA. The hypothalamus-pituitary-ovary axis and type 1 diabetes mellitus: a mini review. Human reproduction. Vol 21. $\mathrm{N}^{\circ}$ 2: 327-337, 2006.

8. The diabetes control and complications trial research group. The effect of intensive treatment of diabetes on the development and progression of long-term complications in insulin-dependent diabetes mellitus. The Diabetes Control and Complications Trial Research Group. The N. Eng. J Med., 329: 977-986, 2003.

9. Bruning JC, Gautam D, Burks DJ, Gillette J, Schubert M, Orban PC, Klein R, Krone W, Muller-Wieland D, Kahn CR. Role of brain insulin receptor in control of body weight and reproduction. Science 289:2122-2125, 2000.

10. La Marca A, Morgante G, De Leo V. Evaluation of hypothalamicpituitary-adrenal axis in amenorrhoeic women with insulin-dependent diabetes. Hum. Reprod. 14:298-302, 1999. 
11. Pal L, Chu HP, Shu J, Topalli I, Santoro N, Karkanias G. In vitro evidence of glucose-induced toxicity in $\mathrm{GnRH}$ secreting neurons: high glucose concentrations influence $\mathrm{GnRH}$ secretion, impair cell viability, and induce apoptosis in the GT1-1 neuronal cell line. Fertil Steril 88:1143-1149,2007.

12. Codner E, Cassorla F. Puberty and ovarian function in girls with type 1 diabetes mellitus. Horm. Res. 71:12-21, 2009.

13. Poretsky L, Cataldo NA, Rosenwaks Z, Giudice LC: The insulinrelated ovarian regulatory system in health and disease. Endocr. Rev. 20: 535-582, 1999.

14. Hsueh AJ, Billig $\mathrm{H}$, Tsafriri A. Ovarian follicle atresia: a hormonally controlled apoptotic process. Endocr. Rev. 15: 707-724, 1994.

15. Codner E, Soto N, López P,Trejo L, Avila A, Eyzaguirre FC, Iniguez G, Cassorla F: Diagnostic criteria for polycystic ovary syndrome and ovarian morphology in women with type 1 diabetes mellitus. J. Clin. Endocrinol. Metab. 91: 2250-2256, 2006.

16. Chang AS, Dale AN, Moley KH. Maternal diabetes adversely affects preovulatory oocyte maturation, development, and granulosa cell apoptosis. Endocrinology 146: 2445-2453, 2005.

17. Diamanti-Kandarakis E, Piperi C, Patsouris E, Korkolopoulou $P$, Panidis D, Pawelczyk L, Papavassiliou AG, Duleba AJ. Immunohistochemical localization of advanced glycation end-products (AGEs) and their receptor (RAGE) in polycystic and normal ovaries. Histochem. Cell Biol. 127:581-589, 2007.

18. Codner E, Eyzaguirre F, Iñiguez G, López P, Perez-Bravo F, Torrealba I, Cassorla F; For the Chilean Group for the Study of Ovarian Function in Type 1 Diabetes. Ovulation rate in adolescents with type 1 diabetes mellitus. Fertility and sterility. Vol. 95, Issue 1, pp. 197-202, 2011

19. Amaral S, Oliveira PJ, Ramalho-Santos J. Diabetes and the impairment of reproductive function: possible role of mitochondria and reactive oxygen species. Current Diabetes Reviews 4: 46-54,2008.

20. Agarwal A, Gupta S, Sharma R. Oxidative stress and its implications in female infertility, a clinician's perspective. Reprod. Biomed. Online 11: 641-50, 2005.

21. Agarwal A, Gupta S, Sharma RK. Role of oxidative stress in female reproduction. Reprod. Biol. Endocrinol. 14: 3-28, 2005.

22. Wilcox AJ, Weinberg CR, Baird DD. Postovulatory ageing of the human oocyte and embryo failure. Hum. Reprod. 13: 394-397, 1998.

23. Nishimi A, Matsukawa T, Hoshino $K$, et al. Localization of nitric oxide synthase activity in unfertilized oocytes and fertilized embryos during pre-implantation development in mice. Reproduction 122: 957-963, 2001.

24. Khorram O. Nitric oxide and its role in blastocyst implantation. Rev. Endocr. Metab. Disord. 3: 145-149, 2002.

25. Merino P, Schulin-Zeuthen C, Codner E. Diagnóstico del síndrome de ovario poliquístico: nuevos fenotipos, nuevas incógnitas. Rev. Med. Chile 137: 1071-1080, 2009.

26. Peppard H, Marfori J, Nestler J et al. Prevalence of polycystic ovary syndrome among premenopausal women with type 2 diabetes. Diabetes Care 24:1050-1052, 2001.

27. Legro R, Kunselman A, William $C$ et al. Prevalence and predictors of risk for type 2 diabetes mellitus and impaired glucose tolerance in polycystic ovary syndrome: a prospective, controlled study in 254 affected women. Journal of Clinical Endocrinology and Metabolism 84 (1): 165-169, 1999.

28. High prevalence of the polycystic ovary syndrome and hirsutism in women with type 1 diabetes mellitus. J. Clin. Endocrinol. Metab. 2000 Nov; 85(11):4182-7.

29. Clinical Review: hyperandrogenism and polycystic ovary syndrome in women with type 1 diabetes mellitus. J. Clin. Endocrinol. Metab., april 2007, 92(4):1209-1216.

30. Teede H, Deeks A, Moran L. Polycystic ovary syndrome: a complex condition with psychological, reproductive and metabolic manifestations that impacts on health across the lifespan. Bio. Med. Central Medicine, 8:41, 2010.
31. Morin-Papunen L et al. JCEM 2012; 97:1492-1500.

32. Hum. Reprod. Update 2005; 11 (4): 375-390.

33. Arturo Vivas, C. Síndrome de ovario poliquístico, endometrio y riesgo de aborto. Revista Colombiana de Obstetricia y Ginecología, 5(4): 303-309, 2005.

34. Ropelato M. Alteraciones neuroendocrinas del síndrome de poliquistosis ovárica en la adolescencia. Revista Argentina de Endocrinología y Metabolismo, 45 (2): 89-110, 2008.

35. PCOS Australian Alliance 2011. A single voice for polycystic ovary syndrome. Evidence based guideline for the assessment and management of PCOS www.jeanhailes.org.au.

36. López J. 2004. Genética en la obesidad. Rev. de Endocrinología y Nutrición. Vol. 12, N 4, Supl. 3.

37. Metwally M, LiTC, Ledger WL. The impact of obesity on female reproductive function. Obes. Rev. 2007; 8:515-23.

38. Ramsay JE, Greer I, Sattar N. ABC of obesity. Obesity and reproduction. BMJ 2006; 333:1159-62.

39. Henry Bohler et al. Fertility and sterility, Vol. 94, N³, august 2010.

40. Cornier MA, Dabelea D, HernandezTL, Lindstrom RC, et al. The metabolic syndrome. Endocr. Rev. 2008; 29:777-822.

41. Ayala Yáñez R, Ayala AR. Adiposidad, inflamación e infertilidad. Revista Mexicana de Medicina de la Reproducción, Vol. 2, N²: 57-62, 2009.

42. Santillana Espinosa E, Avila Esquivel F. Infertilidad e insulinorresistencia, Investigación materno infantil. Vol. I, Nº 3, pp. 118-122, septiembre- diciembre 2009.

43. Pagotto U.Where does insulin resistance start?The brain. Diabetes Care, Vol. 32, Supplement 2, November 2009.

44. Geisthovel F, Jochmann N, Widjaja A, Horn R, Brabant G. Serum pattern of circulating free leptin, bound leptin, and soluble leptin receptor in the physiological menstrual cycle. Fertil Steril 2004; 81:398-402.

45. Cervero A, Horcajadas JA, Mart J, Pellicer A, Simon C. The leptin system during human endometrial receptivity and preimplantation development. J. Clin. Endocrinol. Metab. 2004; 89:2442-51.

46. Zachary $\mathrm{T}$ Bloomgarden. Gut and adipocyte peptides. Diabetes Care, Vol. 29, № 2, February 2006.

47. Nizard Jacky et al. Pregnancy in a woman with a leptin-receptor mutation. August 30, 2012, NEJM, 366;11, 1064-1065.

48. Pasquali Renato et al. Obesity, fat distribution and infertility. Maturitas 2006; 54:363-371.

49. Davelene I, Streamson Chua Jr. Leptin receptor modulation of adiposity and fertility, Endocrinol. Metab. 2011 January; 21

50. Mitchell M, Armstrong DT, Robker RL, Norman RJ. Adipokines: implications for female fertility and obesity. Reproduction 2005;130: 583-97.

51. Delarue J, Magnan C. Free fatty acids and insulin resistance. Curr. Opin. Clin. Nutr. Metab. Care 2007;10:142-8.

52. Bucholz H. J Clin. Endocrinol. Metab. 1999; 84:1470-4.

53. The Practice Committee of the American Society for Reproductive Medicine, Obesity and Reproduction: an educational bulletin. Vol. 90, Suppl. 3, November 2008.

54. Livshits A, Seidman DS. Fertility issues in women with diabetes. Women's Health; 5(6):701-707, 2009.

55. Adcock CJ, Perry LA, Lindsell DR, Taylor AM, Holly JM, Jones J, Dunger DB. Menstrual irregularities are more common in adolescents with type 1 diabetes: association with poor glycaemic control and weight gain. Diabetes Med.; 11:465-470, 1994.

56. Goswami R, Marwaha RK, Goswami D et al. Prevalence of thyroid autoimmunity in sporadic idiopathic hypoparathyroidism in comparison to type 1 diabetes and premature ovarian failure. J. Clin. Endocrinol. Metab.; 91(11), 4256-4259, 2006.

57. Enzlin P, Mathieu C, Van den Bruel A et al. Sexual dysfunction in women with type 1 diabetes: a controlled study. Diabetes Care; 25(4), 672-677, 2002.

58. Dorman JS, Steenkiste AR, Foley TP et al. Menopause in type diabetic women, is it premature? Diabetes 50, 1857-1862, 2001. 
59. Homan G, Davies, M, et al. The impact of lifestyle factors on reproductive performance in the general population and those undergoing infertility treatment: a review. Human Reproduction Update 13: 209-223, 2007.

60. Augood C, Duckitt K, et al. Smoking and female infertility: a systematic review and meta-analysis. Human Reproduction 13: 1532-1539, 1998.

61. Bolumar F, Olsen J. Smoking reduces fecundity: a european multicenter study on infertility and subfecundity. Am. J. Epidemiol. 143: 578- 587, 1996.

62. Agarwal A, Aponte-Mellado A, et al. The effect of oxidative stress on female reproduction: a review reproductive biology and endocrinology 2012, 10:49http://www.rbej.com/content/10/1/49.

63. Tiboni GM, Bucciarelli T, et al. Influence of cigarette smoking on vitamin $E$, vitamin $A$, beta-carotene and lycopene concentrations in human pre-ovulatory follicular fluid. Int. J. Immunopathol. Pharmacol. 17:389-393, 2004.

64. Benedict M, Missmer S, et al. Secondhand tobacco smoke exposure is associated with increase risk ok failed implantation and reduced IVF success. Human. Reproduction, 26: 2525-2531, 2011.

65. Howe, G Westhoff $C$, et al. Effects ok age, cigarette smoking and other factors on fertility: findings in a large prospective study. BMJ 290: 1697, 1985.

66. Tolstrup JS, Kjaer SK, Holst C, et al. Alcohol use as predictor for infertility in a representative population of danish women. Acta Obstet. Gynecol. Scand. 82:744-749, 2003.

67. Hakim R, Gray R, et al. Alcohol and caffeine consumption and decreased fertility. Fertil Steril 70: 632-637, 1998.

68. Kumar S. Occupational, environmental and lifestyle factors associated with spontaneous abortion. Reprod. Sci. 18:915-930, 2011.

69. Cicero AF, Tartagni E, Ertek S. Metformin and its clinical use: new insights for an old drug in clinical practice. Free Arch. Med. Sci. 2012 Nov 9; 8(5):907-17

70. Motta DA. Metformin in the treatment of polycystic ovary syndrome. Curr. Pharm. Res. 2008; 14: 2121-5.

71. Xiao J, Chen S, Zhang C, Chang S. The effectiveness of metformin ovulation induction treatment in patients with PCOS: a systematic review and meta-analysis. Gynecol. Endocrinol. 2012 Dec; 28(12):956-60.

72. Tomova A, Deepinder F, Robeva R, Kirilov G, Mechandjiev Z, Kumanov P. Anti-müllerian hormone in women with polycystic ovary syndrome before and after therapy with metformin. Horm. Metab. Res. 2011 Sep; 43(10):723-7.

73. Tang T, Lord JM, Norman RJ, Yasmin E, Balen AH. Cochrane Database Syst. Rev. 2012 May.

74. Sinawat $S$, Buppasiri $P$, Lumbiganon $P$, Pattanittum P. Long versus short course treatment with metformin and clomiphene citrate for ovulation induction in women with PCOS. Cochrane Database Syst. Rev. 2012 Oct 17;10.

75. Teede H, Misso M, Deeks A, et al. 2011. MJA 195 (6) S 65-112.

76. Palomba S, Falbo A. La Sala GB BJOG. 2013 Feb; 120(3):267-76.

77. Xiao J, Chen S, Zhang C, Chang S. Gynecol. Endocrinol. 2012 Dec; 28(12):956-60.

78. Palomba S, Falbo A, Orio F. Fertility and sterility. 2009; 92(5):1646-1658.

79. Duranteau L, Lefevre P, Jeandidier N, Simon T, Christin-Maitre S. Should physicians prescribe metformin towomen with polycystic ovary syndrome (PCOS)? Ann. Endocrinol. 2010; 71: 25-7.

80. Rouzi AA, Ardawi MS. A randomized controlled trial of the efficacy of rosiglitazone and clomiphene citrate versus metformin and clomiphene citrate in women with clomiphene citrate-resistant polycystic ovary syndrome. Fertil Steril. 2006 Feb; 85(2):428-35.
81. Minge CE, Robker RL, Norman RJ. PPAR Gamma: coordinating metabolic and immune contributions to female fertility. PPAR Res. 2008:243791.

82. Tang T, Lord JM, Norman RJ, Yasmin E, Balen AH. Insulin-sensitising drugs (metformin, rosiglitazone, pioglitazone, D-chiroinositol) for women with polycystic ovary syndrome, oligo amenorrhoea and subfertility. Cochrane Database Syst. Rev. 2012, May 16; 5:CD003053.

83. Fromentand $P$, Touraine P.Thiazolidinediones and fertility in polycystic ovary syndrome (PCOS). PPAR Research, Vol. 2006, article ID 73986,1-8.

84. ElhaddT, FiadT, Meer L. Ovarian stockpiling in polycystic ovary syndrome, infertility, and the combined use of rosiglitazone and metformin. Diabetes Care, 2006. 29: 2330-2331.

85. Ota H, Goto T, Yoshioka T, Ohyama N. Successful pregnancies treated with pioglitazone in infertile patients with polycystic ovary syndrome. Fertil Steril. 2008 Sep; 90(3):709-13.

86. Kircher C, Smith KP. Acarbose for polycystic ovary syndrome. Ann Pharmacother. 2008 Jun; 42(6):847-51.

87. Ciotta L, Calogero A, Farina M, De Leo V, La Marca A, Cianci A. Clinical, endocrine and metabolic effects of acarbose, and aglucosidase inhibitor, in PCOS patients with increased insulin response and normal glucose tolerance. Hum. Reprod. 2001; 16:2066-72.

88. Penna IAA, Canella PRB, Reis RM, Silva de Sá MF, Ferriani RA. Acarbose in obese patients with polycystic ovarian syndrome: a double-blind, randomized, placebo-controlled study. Hum Reprod 2005;20:2396-401.

89. Tugrul S, Kutlu T, Pekin O, Baglam E, Kiyak H, Oral O. Clinical, endocrine, and metabolic effects of acarbose, a alpha-glucosidase inhibitor, in overweight and nonoverweight patients with polycystic ovarian syndrome. Fertil Steril. 2008 Oct; 90(4):1144-8.

90. Sönmez AS, Yasar L, Savan K, et al. Comparison of the effects of acarbose and metformin use on ovulation rates in clomiphene citrate-resistant polycystic ovary syndrome. Hum. Reprod. 2004; 20:175-9.

91. Gaal Z, Klupa T, Kantor I, Mlynarski W, Albert L, Tolloczko J, Balogh I, Czajkowski K, Malecki MT. Sulfonylurea use during entire pregnancy in diabetes because of KCNJ11 mutation: a report of two cases. Diabetes Care. 2012 Jun; 35(6):e40.

92. KlupaT, Kozek E, Nowak $\mathrm{N}$ et al. The first case report of sulfonylurea use in a woman with permanent neonatal diabetes mellitus due to KCNJ11 mutation during a high-risk pregnancy. J. Clin. Endocrinol. Metab. 2010 Aug; 95(8):3599-604.

93. Tieu J, Coat S, et al. Oral anti-diabetic agents for women with pre-existing diabetes mellitus/impaired glucose tolerance or previous gestational diabetes mellitus. Cochrane Database of Systematic Reviews. In: The Cochrane Library, Issue 7, Art. N CD007724.

94. Strauss TJ, Castrillon DH, Hammes SR. GATA-like protein-1 (GLP1 ) is required for normal germ cell development during embryonic oogenesis. Reproduction. 2011 Feb; 141(2):173-81.

95. Rüter J, Hoffmann T, Heiser U, Demuth HU, Arck PC, Klapp BF, Hildebrandt M. The expression ofT-cell surface antigens CTLA-4, CD26, and CD28 is modulated by inhibition of dipeptidylpeptidase IV (DPP IV, CD26) activity in murine stress-induced abortions. Cell Immunol. 2002 Dec; 220(2):150-6.

96. Nishikawa M, Itakura A, Ito M, Takeuchi M, Sato $Y$, Kajiyama $H$, Mizutani S, Kikkawa F. Changes in placental dipeptidyl peptidase IV in preeclampsia with intrauterine growth restriction. Horm. Metab. Res. 2005 Jul; 37(7):408-13. 